2014-5

\title{
A Miniaturized Flexible Surface Attachable Interrogator for Hybrid Optical Fiber Sensing
}

\author{
Yuliya Semenova \\ Technological University Dublin, yuliya.semenova@tudublin.ie \\ Manjusha Ramakrishnan \\ Technological University Dublin, manjusha.ramakrishnan@mydit.ie \\ Ginu Rajan \\ The University of New South Wales
}

See next page for additional authors

Follow this and additional works at: https://arrow.tudublin.ie/prcart

Part of the Electrical and Computer Engineering Commons

\section{Recommended Citation}

Ramakrishnan, M. et al. (2014) A Miniaturized Flexible Surface Attachable Interrogator for Hybrid Optical Fiber Sensing, MICROWAVE AND OPTICAL TECHNOLOGY LETTERS Vol. 56, no. 5, pp. 1167-1174, May. doi:10.1002/mop.28284

This Article is brought to you for free and open access by the Photonics Research Centre at ARROW@TU Dublin. It has been accepted for inclusion in Articles by an authorized administrator of ARROW@TU Dublin. For more information, please contact arrow.admin@tudublin.ie, aisling.coyne@tudublin.ie,gerard.connolly@tudublin.ie.

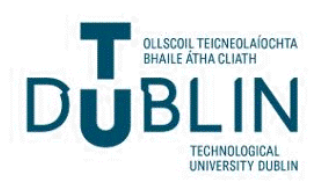




\section{Authors}

Yuliya Semenova, Manjusha Ramakrishnan, Ginu Rajan, Tomasz Wolinski, Andrzej Domański, and Gerald Farrell 


\title{
A Miniaturized Flexible Surface Attachable Interrogator for Hybrid Optical Fiber Sensing
}

\author{
Manjusha Ramakrishnan $^{{ }^{*}}$, Ginu Rajan ${ }^{2}$, Yuliya Semenova ${ }^{1}$, Tomasz Wolinski ${ }^{3}$, \\ Andrzej Domański ${ }^{3}$ and Gerald Farrell ${ }^{1}$ \\ ${ }^{1}$ Photonics Research Centre, Dublin Institute of Technology, Dublin, Ireland \\ ${ }^{2}$ School of Electrical Engineering and Telecommunications, \\ The University of New South Wales, Sydney, Australia \\ ${ }^{3}$ Faculty of Physics, Warsaw University of Technology, Warsaw Poland \\ *Email: manjusha.ramakrishnan@mydit.ie
}

\begin{abstract}
In this paper, we propose a miniaturized flexible interrogator for polarimetric and fiber Bragg grating (FBG) sensors based hybrid sensing scheme embedded in composite materials. The flexible interrogation module comprises of an Arrayed Waveguide (AWG) for FBG demodulation and an Electro Optic Mach- Zehnder Interferometric (EO-MZI) intensity modulator for polarimetric sensor demodulation. A customized design of AWG and EO- MZI are presented, and the designed performance resulted in a wide channel spacing of $5 \mathrm{~nm}$ and low cross talk of -34 dB between adjacent channels for the AWG, and a low V $\pi$ voltage of \pm 1.5 Volt for the MZI-EO. The method for fabrication of the flexible interrogator device is also presented, based on post processing of platinum coated polyimide substrate. The film type interrogation module is compact, with a size of $3.4 \mathrm{~cm} \times 0.1 \mathrm{~cm} \times 0.01 \mathrm{~cm}$. The surface attachable flexible hybrid sensor interrogator is proposed to integrate with photo detector arrays and wireless communication technology to enhance the competency of hybrid sensing scheme in smart sensing composite parts in motion for applications in aircraft, wind rotor blades, and so forth.
\end{abstract}




\section{INTRODUCTION}

The use of fiber optic sensing based structural health monitoring (SHM) [1] for composite structures is well established in applications such as aerospace, infrastructure, marine, wind rotor blades, etc. The interrogation systems traditionally used in field applications of optical fiber sensors are too bulky and cumbersome for applications in composite structures and also are difficult to package. In comparison the integrated optoelectronic devices [2], commonly found in optical communications systems are significantly smaller and easier to package. For several sensing applications, such as fiber Bragg gratings (FBGs) systems and fiber optic gyros (FOGs) [3], interrogation systems based on integrated optical devices have been reported [4]. For example, integrated Arrayed Waveguides [4] (AWGs) / bandpass wavelength filters are extensively employed as the core of FBG interrogation systems, while integrated optic based Electro-Optic (EO) modulators [3] have been shown to be suitable for FOG demodulation purposes.

Sensing for composite materials in some aerospace components and other applications such as wind turbines will frequently involve embedding fiber sensors in parts which are in constant motion. In such applications fiber based connections to an interrogation system mounted remotely are difficult to implement. An alternative is the use of a compact interrogator mounted on the composite part itself which utilizes wireless system to communicate to the end user. For such applications, the ability to attach an integrated interrogation system to the surface of the composite would be an advantage. For this a flexible rather than rigid waveguides and devices are more suitable. Flexible optical waveguides $[5,6]$ with embedded thin-film vertical-cavity surface-emitting lasers and photo detector arrays have been proposed as solutions to the packaging challenges of large-scale integration for telecommunication applications. However the potential of such flexible integrated optic devices for optical fiber sensor interrogation purposes has not been fully realized yet.

In this paper we propose a miniaturized flexible interrogation module for a hybrid sensing [7] scheme which can be attached as a "table top" component to a surface of a composite structure. The hybrid sensor is comprised of two types of fiber sensors: a high-birefringence polarization maintaining fiber based polarimetric sensor [8] combined with an FBG sensor. Using the hybrid sensor approach, simultaneous 
measurement of strain and temperature in composite materials has been reported earlier [9]. This involves converting the polarimetric and FBG sensors outputs into intensity domain using edge filters. In our previous paper [10], an experimental demonstration of the hybrid sensor interrogation module is realised using a commercially available Thin Film Filter (TFF) based WDM demultiplexer for FBG demodulation and a Lithum Niobate phase modulator for interrogation of polarimetric sensors. The working principle of the flexible AWG based FBG interrogator proposed in this paper is similar to that in [10]. In principle it is possible to use off-the-shelf dense WDM devices, designed for telecommunication applications, with channel spacing in the order of $1 \mathrm{~nm}$ or less for interrogation of FBG sensors. The limitation of such off-the-shelf devices for this application is their narrow channel spacing, so that a complex algorithm is needed to extract FBG peak shift information. Thus in the flexible interrogation scheme proposed here, the AWG is designed with a wide channel spacing of $5 \mathrm{~nm}$ and a small cross talk value of $-34 \mathrm{~dB}$ between adjacent channels. For a polarimetric sensor, an external perturbation leads to a phase change and an electro-optic phase modulator is can be utilized for polarimetric sensors interrogation [10]. In this paper we report for the first time the design and fabrication of a miniaturized flexible interrogation module for a hybrid sensing system for composite materials.

There have been previous reports of flexible waveguide based AWGs [11], fabricated by soft moulding of Polydimethylsiloxane (PDMS). However the processing of PDMS is quite difficult. The fabrication of flexible electro-optic polymer based EO devices by the post lift-off method was reported earlier [12], in which a fabricated device is released from a wafer by utilizing the low adhesive property of SU-8 polymer on gold. In this paper, the proposed flexible interrogation module is designed based on two polymer waveguide based devices [13], an AWG and a Mach-Zehnder interferometric electro-optic (MZI-EO) intensity modulator. The core/cladding polymers of both the MZI-EO device and AWG are selected to facilitate a flexible waveguide based device fabrication. The AWG is based on a Cyclotene polymer as the core material, while the MZI-EO device utilizes an EO polymer AJL146 [14] as the core material.

The paper is organized as follows. The hybrid sensor methodology is described in section 2. The detailed explanation of the proposed flexible interrogation module is 
provided in section 3. The simulated performance and proposed fabrication process of the flexible sensor interrogation module are presented in section 4 . The fabricated interrogator system integration possibilities are discussed in section 5.

\section{HYBRID SENSOR - METHODOLOGY}

In a polarimetric sensor a symmetric deformation effect in a single-mode fiber influences the propagation constant $(\beta)$ of every mode, which leads to changes in the phase difference between both polarizations of the fundamental mode LP01 [8]. The phase difference between orthogonal polarizations, induced by the polarimetric sensor, results in a sinusoidal intensity variation, when the sensor is placed in crossed polarizers. There are different varieties of polarimetric sensors [8] involving different types of high-birefringence polarization maintaining fibers. The temperature and strain sensitivity of each of the polarimetric sensor types is different. In the case of a Panda fiber, both strain and temperature changes lead to a phase difference $[8,9]$, while in the case of a PM-PCF the phase difference originates only from longitudinal strain and has a very low sensitivity to temperature [7].

While polarimetric sensors can provide average strain measurements, they are not suitable for point strain measurements, which are also frequently required. For such measurements, FBGs are commonly employed, but with the problem that the Bragg wavelength changes with both strain and temperature. By employing a hybrid sensing approach, where both FBG and polarimetric sensors are used, the cross-sensitivity issue of temperature for FBG strain measurements can be eliminated, using the temperature independent strain information from the polarimetric sensors. The average strain and temperature in the sample can also be measured either by comparing the average strain from different types of polarimetric sensors or by taking the average of localized point strains. More details on the simultaneous measurement of average and local strain and temperature in composite materials are discussed in our previous research paper $[7,9]$. 


\section{THE FLEXIBLE INTERROGATION MODULE}

The experimental setup for a hybrid sensor system and the proposed interrogation demonstration can be realized as shown in Figure 1, in which for illustration purposes the case of five FBG sensors and two polarimetric sensors is shown. The setup comprises of a compact distributed feedback laser (working in the wavelength range from $1530 \mathrm{~nm}$ to $1570 \mathrm{~nm}$ ), a fiber optic in-line isolator, a polarization maintaining (PM) 1 x 3 optical fiber splitter, $1550 \mathrm{~nm}$ wavelength filters (for polarimetric sensors), an in-line fiber optic polarizer and analyzer, the polarimetric sensors (Panda and PM-PCF), an in- line fiber optic circulator, an FBG array, a flexible hybrid sensor interrogation module (combining the MZI-EO intensity modulator and AWG), an array of photo receivers. The outputs of the receivers could be connected in turn via a wireless link to a data processing unit.

We have previously demonstrated [10] that it is possible to interrogate both FBGs and polarimetric sensors in the intensity domain using wavelength dependent edge filters for converting the wavelength changes from FBGs and using EO modulators for converting the phase changes induced by polarimteric sensors into intensity variations. In such scheme wavelength information from an array of FBGs can be easily converted into intensity domain using an AWG whose multiple input wavelengths are designed to match the Bragg wavelengths of the FBG sensors [4, 10]. For interrogation of a polarimetric sensor, its output is connected to the input arm of an MZI electro-optic intensity modulator, the operating voltage of which is chosen in such a way that any change in the output from the polarimetric sensor causes a linear variation of the modulator's output intensity. As a result any external perturbation of the polarimetric sensor (e.g., strain) is easily converted into linear changes in the output intensity.

It should be noted that a single MZI EO device can be used for interrogation of multiple polarimetric sensors simultaneously using time domain multiplexing. The response of multiple polarimetric sensors can be separated in time domain by appropriate processing of the detector output. The polarimetric sensors used can be based on a wide variety of highly birefringent polarization maintaining fibers such as, PANDA fibers, side holed and micro-structured PM-PCF. 


\section{DESIGN AND FABRICATION OF THE PROPOSED FLEXIBLE INTERROGATOR}

When implemented as a flexible interrogator, both the MZI-EO device and the AWG are polymer-based optical waveguide devices. The polymer materials for core and cladding of the flexible interrogator are selected based on their adhesion properties and compatibility with existing integrated - optic / CMOS fabrication facilities.

For fabrication of the proposed flexible interrogator a method called the post-lift off method is adopted, in which the low adhesion between polyimide and a layer of platinum is utilized to lift the fabricated device off a silicon wafer used as a base only. Both the MZI-EO device and AWG are fabricated on a common polyimide substrate [15] film. A $100 \mu \mathrm{m}$ thick polyimide layer is flexible and strong enough to support the MZI-EO device and AWG devices. The polyimide substrate also offers better thermal stability due to its good thermal properties [16].

\subsection{Design and simulation of a flexible 1x6 Arrayed Waveguide}

In this section we present the design of a 1x6 channel AWG and its performance optimization achieved using OptiBPM [17] and WDM Phasar tools. The optimized AWG is intended for the purpose of FBG sensors interrogation. In the example here a six-channel AWG is designed to suit the interrogation of six FBGs (with matching peak wavelengths). The key requirements while optimizing the design of this $1 \mathrm{x} 6$ AWG are: a wavelength range of $1535 \mathrm{~nm}$ to $1565 \mathrm{~nm}$; a wide channel spacing of 5 nm over this wavelength range, with low crosstalk between adjacent AWG channels. Each channel of such of an AWG can be used to convert a Bragg wavelength shift of up to $2.5 \mathrm{~nm}$ for a single FBG sensor into the intensity domain.

The AWG is designed based on UV curable polymers Cyclotene 3022-46 (Microchem) with an RI of 1.535 as the core material and Ormoclad (Microchem) with an RI of 1.522 as the upper/ lower cladding material. Single mode wave propagation is achieved using a rib waveguide structure with a rib height of $4 \mu \mathrm{m}$, width of $4 \mu \mathrm{m}$ and etch height of $1 \mu \mathrm{m}$ as shown in Figure 2. The designed rib waveguide structure offers low propagation loss $<0.05 \mathrm{~dB} / \mathrm{cm}$. 
The design parameters used for the custom design the AWG geometry are as follows: the wavelength spacing between AWG channels, center wavelength, and effective index of waveguide are $5 \mathrm{~nm}, 1.5425$, and 1.5330241, respectively. The custom designed AWG [18] layout is as shown in Figure 3. The operation of the AWG device is explained in [18] as follows. When a mode in the input waveguide enters the input Free Spectral Region (FSR) it becomes divergent. In the phased array (PA) region a spatial separation of different wavelengths occurs by introducing a length difference between adjacent PA waveguides. Further, a wavelength-dependent angular tilt occurs so the outgoing modes at different wavelengths are coupled to different output ports using the output side FSR.

In order match typical commercial fiber dimensions, the gap between the adjacent output channels of the AWG is set to $250 \mu \mathrm{m}$ as shown in Figure 3. The geometrical parameters of the custom designed AWG are optimized to achieve the performance specification mentioned earlier. The final geometrical parameters of the custom designed AWG are as follows: the number of waveguides in the PA region is 26, the length increment of PA waveguides is $26.254 \mu \mathrm{m}$, the PA waveguide separation is $27.107 \mu \mathrm{m}$, the minimum distance between waveguides in the PA region is $20 \mu \mathrm{m}$, the FSR regions lengths are $4352 \mu \mathrm{m}$. The AWG flexible film size is $34 \times 6.4 \mathrm{~mm}$. A Gaussian shaped channel response is selected for the AWG and the optimized optical transmission of the AWG for TE polarization is as in Figure 4.

Simulation using WDM Phasar shows that the optimized design AWG offers a wide channel spacing of $5 \mathrm{~nm}(625 \mathrm{GHz})$ between six channels located at $1532 \mathrm{~nm}$, $1537 \mathrm{~nm}, 1542 \mathrm{~nm}, 1547 \mathrm{~nm}, 1552 \mathrm{~nm}$ and $1557 \mathrm{~nm}$, with adjacent channel crosstalk value of $-34 \mathrm{~dB}$. For an input of $0 \mathrm{dBm}$ to the $\mathrm{AWG}$ the insertion loss is estimated to be less than $10 \mathrm{~dB}$.

\subsection{Design and simulation results for a flexible EO Intensity Modulator}

In this section we present the design of an MZI-EO and results of its performance optimization simulated using Optimode solver and OptiBPM tools. The optimized MZI EO is intended for the purpose of polarimetric fiber sensor interrogation. The key requirements in optimizing the MZI-EO design are that the waveguide should support single mode operation with low propagation loss and a low V $\pi$ voltage. 
The MZI-EO is designed with a guest-host EO polymer AJ416 [14] as the core material, with refractive index of 1.6403 for the TE mode. The reason for selecting the AJ416 polymer as a core material is its key benefits such as high thin film $\gamma 33$ value of $120-130 \mathrm{pm} / \mathrm{V}$, and high stability $[14,19]$. A UV-curable resin Cyclotene 3022-46 with an RI of 1.535 is chosen as the upper/ lower cladding.

In the MZI EO device single mode operation is optimized using an oversized waveguide structure [19]. It has been proved in earlier research works that the singlemode condition does not require a thin core layer. Even if the core layer is thick so that it could in principle support several modes in the slab waveguide, it is still possible to design the rib waveguide so as to support only one mode by radiating the higher order modes horizontally into the slab modes [19]. For optimizing the rib height for single mode operation, the TE refractive indices of the fundamental $\left(0^{\text {th }}\right.$ order) and higher order modes $\left(1^{\text {st }}\right.$ order and $2^{\text {nd }}$ order) are calculated using the Optimode solver tool for planar waveguides with a $4 \mu \mathrm{m}$ width for various thicknesses of core layer as shown in Figure 5. For the fundamental mode to propagate through the rib region the effective index contrast between the entry rib and side slab sections is $\Delta \mathrm{n}_{\mathrm{eff}}=0.003$ [19]. From Figure 5, it is obvious that for a $3 \mu \mathrm{m}$ core thickness, the etch depth of $\Delta \mathrm{T}=0.5 \mu \mathrm{m}$ results in an effective index difference of 0.003 . The effective index of the $1^{\text {st }}$ order TE mode for a $3 \mu \mathrm{m}$ thick center rib region is less than ( $\mathrm{n}_{1 \text { center }}<\mathrm{n}_{0 \text { center }}$ ) the effective index of the fundamental mode in a $2.5 \mu \mathrm{m}$ slab region. Consequently, the higher order modes propagation through the slab region changes to a single mode operation within the rib waveguide region.

The inverted rib-waveguide structure is adopted for the MZI -EO modulator with a $4 \mu \mathrm{m}$ width, $3 \mu \mathrm{m}$ rib height and $0.5 \mu \mathrm{m}$ etch height as shown in Figure 6. The key benefit of such an inverted-rib structure [20] is that the rib is processed in the robust cured lower cladding region instead of the core layer, thus reducing the processing requirements for the core layer.

The 2x2 MZI EO intensity modulator design is initially laid out using the OptiBPM waveguide layout designer tool and simulation is performed at $1.55 \mu \mathrm{m}$ wavelength using a beam propagation method [17].The MZI EO design consists of a $3 \mathrm{~dB}$ splitter, electrode interaction region and a $3 \mathrm{~dB}$ combiner as shown in Figure 7(a). Both the $3 \mathrm{~dB}$ splitter and $3 \mathrm{~dB}$ combiner are identical and have patterns that are mirror images of each other with a length of $6.5 \mathrm{~mm}$. The $3 \mathrm{~dB}$ splitter/combiner consists of a linear 
waveguide, an S bend, coupling linear waveguide and a second $\mathrm{S}$ bend region; the length of each region is as shown in Figure 7(b). The $S$ bend dimensions are chosen to reduce the losses during switching.

Using the Electro optic solver, the phase change of the optical field with applied voltage is simulated. It is observed that a phase change of $-\pi / 2$ occurs when an electrode voltage of - 1.5 Volt is applied to the linear waveguide with electrode interaction length of $1 \mathrm{~cm}$, as shown in the Figure 8. Further, in our MZI-EO device the electrode configuration is push-pull [12], achieved by applying equal and opposite voltages at the two electrode arms of MZI-EO. The push-pull configuration of the MZI-EO device gives maximum possible intensity change for a minimum applied voltage at the electrodes.

For the designed MZI-EO device by applying a $+1.5 \mathrm{~V}$ to the MZI arm 1 and $-1.5 \mathrm{~V}$ to the MZI arm 2 as shown in Figure 7(a), the optical field at the output port $\mathrm{P}_{2}$ switches from minimum to maximum. The simulated amplitude at the output port $\mathrm{P}_{2}$ of the MZI-EO device, with corresponding electrode voltages is shown in Figure 9.

It is obvious that the $\mathrm{V} \pi$ voltage is $\pm 1.5 \mathrm{~V}$. The total chip size of the optimized MZIEO device is $23 \mathrm{~mm} \times 0.1 \mathrm{~mm}$, where the electrode interaction regions have a length of $1 \mathrm{~cm}$. The MZI-EO is optimized to achieve the lowest propagation loss of $0.07 \mathrm{~dB} / \mathrm{cm}$.

\subsection{Proposed fabrication method for the flexible AWG and MZI EO devices}

The flexible AWG and MZI-EO are fabricated by the post lift-off method. The AWG/ MZI-EO device structures are processed on the flexible polyimide substrate. Polyimide does not adhere well to platinum [15] and this property is utilised for the peeling of the device film. The Polyimide substrate with the fabricated AWG/ MZIEO can thus be released from the wafer when processing is completed. The actual fabrication of AWG and MZI-EO are very similar, for example the method of patterning of the substrate layer and peeling off the device. Further, the proposed device fabrication method is simpler, and is compatible with existing facilities for complementary metal-oxide-semiconductor (CMOS) [21] fabrication. The flexible device fabrication process involves four steps. The releasing layer and the flexible polyimide substrate patterning, the device fabrication, the dicing and the device lift 
off. The detailed description of these steps for fabrication of the AWG / MZI-EO is as follows:

Steps in the fabrication of the flexible AWG

3.3.1 Releasing layer and the flexible polyimide substrate patterning:

The first step in fabrication is flexible polyimide substrate patterning on a silicon wafer. If a releasing platinum layer is placed in between silicon wafer and polyimide flexible substrate, it is possible to peel off the device after fabrication. However, the flexible polyimide substrate requires a strong base to firmly hold the device during the robust fabrication process. This is ensured by arranging that rectangle corners at the edges of the silicon wafer are left without the platinum layer and thus the platinum releasing layer needs to be sputtered with such a rectangular lift off mask pattern. On top of the platinum releasing layer the $100 \mu \mathrm{m}$ thick polyimide substrate layer is spin coated. This step is shown in Figure 10(a).

\subsubsection{AWG device fabrication:}

On top of the polyimide substrate layer the $2.5 \mu \mathrm{m}$ thick lower cladding material layer (Ormoclad) is spin coated. On top of the lower cladding layer the $4 \mu \mathrm{m}$ thick core material layer (Cyclotene) is spin coated. Further, with the help of an arrayed waveguide mask pattern, the AWG is patterned on the core layer. The rib waveguide structure of AWG is processed using reactive ion etching (RIE) to achieve a $1 \mu \mathrm{m}$ etch height. Again, on top of the core material layer (Cyclotene), the $2.5 \mu \mathrm{m}$ thick upper cladding material layer (Ormoclad) is spin coated. This step is shown in Figure 10(b).

\subsubsection{Dicing:}

From the four sides, the silicon wafer is diced so as to remove the edges of the silicon layer that are left un-patterned (to hold the device) to facilitate release. This step is shown in Figure 10(c).

\subsubsection{Device lift off:}

The last and final step is device lift off from silicon wafer utilising the low adhesive property of platinum with polyimide. This step is shown in Figure 10(d).

\section{Steps for fabrication of the flexible MZI-EO}

The fabrication steps are as shown in Figure 11. Since the steps involved in the fabrication of MZI-EO device are similar to that of AWG device fabrication, (such as 
the releasing layer and the flexible polyimide substrate layer patterning, the dicing and the device lift off) these steps are not explained in this section. However, the patterning of the MZI-EO device and the platinum electrode layer are dissimilar to that of AWG device and these steps are explained as follows.

In the case of the MZI-EO device, the platinum bottom electrode needs to be patterned on top of the polyimide substrate layer. However, the low adhesion of this bottom platinum electrode layer to the polyimide substrate layer can lead to detachment of the flexible film MZI-EO device from the polyimide substrate layer. Thus, to ensure firm binding of the MZI-EO device on top of the flexible polyimide substrate, the bottom electrode is patterned so that points on the bottom electrode act as anchors for the MZI-EO device.

MZI-EO device fabrication: On top of the patterned bottom electrode, a $2.5 \mu \mathrm{m}$ thick layer of Cyclotene is spin coated to form the lower cladding. Through the partial RIE the mask of the MZI-EO device is patterned on the lower cladding layer to achieve $0.5 \mu \mathrm{m}$ etch height. Since the MZI- EO device is designed with the inverted rib structure, processing is performed on the lower cladding. This reduces processing of the core layer. On top of the lower cladding layer the $3 \mu \mathrm{m}$ thick core material layer (EO polymer AJL416) is spin coated. Again, on top of the core material layer a $2.5 \mu \mathrm{m}$ thick upper cladding layer (Cyclotene) is spin coated. Finally on top of the Cyclotene the upper cladding platinum top electrode is patterned as shown in Figure 11(c).

\section{SYSTEM INTEGRATION CONSIDERATIONS}

The flexible interrogation module is in the process of being fabricated, having a size of $3.4 \mathrm{~cm} \times 0.1 \mathrm{~cm} \times 0.01 \mathrm{~cm}$. As an example of current progress the first sample of the interrogation module is shown in Figure 12(a), appearing as a thin film device. A further example is the polymer rib waveguides within the thin film module, shown in Figure 12(b) as a SEM image of the rib structure of the AWG device. It is clear that flexible film interrogator is light weight and miniaturized. Such a surface attachable interrogator has the potential not to add to the mechanical loading of composite structures. 


\section{Conclusion}

A miniaturized flexible surface attachable interrogator has been proposed for polarimetric and FBG sensors based hybrid sensing scheme embedded in composite materials. The designed interrogator comprised of two polymer waveguide based devices: an AWG for FBGs demodulation and an EO- MZI intensity modulator for polarimetric sensor demodulation. The simulated performances of the designed AWG and EO- MZI resulted in a wide channel spacing of $5 \mathrm{~nm}$ and low cross talk of -34 dB between adjacent channels for the AWG, and a low V $\pi$ voltage of \pm 1.5 Volt for the MZI-EO. The proposed interrogator has been realized as a flexible device using post processing of platinum coated polyimide substrates. The fabricated flexible film type interrogation module is compact, with a size of only $3.4 \mathrm{~cm} \times 0.1 \mathrm{~cm} \times 0.01 \mathrm{~cm}$. Additionally, it has been proposed to integrate the surface attachable flexible hybrid sensor interrogator with photo detector arrays and wireless communication technology to enhance the application of hybrid sensing scheme in smart sensing and SHM of composite parts in motion for applications in aircraft, wind rotor blades, so forth.

\section{ACKNOWLEDGMENT}

This work was supported by Enterprise Ireland under the international research grant MATERA ERA-NET.

\section{REFERENCES}

[1] E J Friebele, et al.,"Optical fiber sensors for spacecraft applications", Smart Mater. Struct. 8, 813-838, 1999.

[2] Won T. Tsang et al.,"Integrated Opto-electronics: Practicalities and Potentials", Proc. of SPIE Vol. 5624.

[3] Feng Sun, et al., "Study on the Drift of Modulated Phase in Interference Fiber Optic Gyroscope", Journal Of Computers, Vol. 5, No. 3, March 2010.

[4] Y. Sano and T. Yoshino, "Fast optical wavelength interrogator employing arrayed waveguide grating for distributed fiber Bragg grating sensors,” J. Lightwave Technol. 21, 132-139, 2003. 
[5] Chulchae Choi, et al, „Flexible Optical Waveguide Film Fabrications and Optoelectronic Devices Integration for Fully Embedded Board-Level Optical Interconnects”, Journal Of Lightwave Technology, Vol. 22, No. 9, September 2004.

[6] E. Bosman, "Optical interconnections embedded in flexible substrates", Proceedings Symposium IEEE/LEOS Benelux Chapter, Eindhoven, 2006.

[7] Ginu Rajan, et al.,"A Photonic Crystal Fiber and Fiber Bragg Grating-Based Hybrid Fiber-Optic Sensor System", IEEE Sensors Journal, Vol. 12, No. 1, January 2012.

[8] T. R. Wolinski, P. Lesiak, and A. W. Domanski, "Polarimetric optical fiber sensors of a new generation for industrial applications,” Bull. Pol. Ac.: Tech., vol. 56, no. 2, pp. 125-132, 2008.

[9] Rajan G, et al., "A hybrid fiber optic sensing system for simultaneous strain and temperature measurement and its applications”, Photonics Letters Of Poland, vol. 2 (1) pp 46-48, 2010.

[10] M Ramakrishnan, et. al., "A demodulation scheme for a hybrid fiber sensor system for composite materials", Proc. of SPIE, Vol. 8439, SPIE doi:10.1117/12.922627, 2012.

[11] Jung-Gyu Lim, et. al, "Polymeric Arrayed Waveguide Grating Using Imprint Method Based on a Flexible PDMS Stamp" IEEE Xplor, Digital Object Identifier : 10.1109/LEOS.2006.278751, Oct. 2006.

[12] Hyun-Chae Song, et al, “ Flexible low-voltage electro-optic polymer modulators”, Patent no: US 7,366,363,B290089-048,2008.

[13] Hong Ma et. al., "Polymer based optical waveguides: materials, processing, and devices"Adv. Mater. 14, No. 19, 1339-1365, 2002.

[14] Diyun Huang et. al, "Highly efficient EO polymers for low V $\pi$ modulators", Linear and Nonlinear Optics of Organic Materials VIII, Proc. of SPIE Vol. 7049, 70490H, doi: 10.1117/12.795561, 2008.

[15] S.Y. Xiao, "A novel fabrication process of MEMS devices on polyimide flexible substrates", Microelectronic Engineering 85, 452-457, 2008.

[16] Farah, John, "Polished polymide substrate", United States Patent 6563998, 2003.

[17] OptiBPM, Waveguide optics modeling software system, version 8.0, second edition, Optiwave Inc. 2006. 
[18] Meint K. Smit, " PHASAR-Based WDM-Devices: Principles, Design and Applications", IEEE Journal Of Selected Topics In Quantum Electronics, Vol. 2, No. 2, June 1996.

[19] Min-Cheol Oh, et al, "Recent Advances in Electrooptic Polymer Modulators Incorporating Highly Nonlinear Chromophore” IEEE Journal On Selected Topics In Quantum Electronics, Vol. 7, No. 5, September/October,2011

[20] Seong-Ku Kim, et al, Inverted-Rib Waveguide Structure, Ieee Photonics Technology Letters, Vol. 15, No. 2, February 2003.

[21] O. Brand, "Fabrication Technology" Advanced Micro and Nanosystems. Vol. 2. CMOS - MEMS, WILEY-VCH Verlag GmbH \& Co. KGaA, Weinheim, ISBN: 3527-31080-0, 2005. 


\section{Figures}

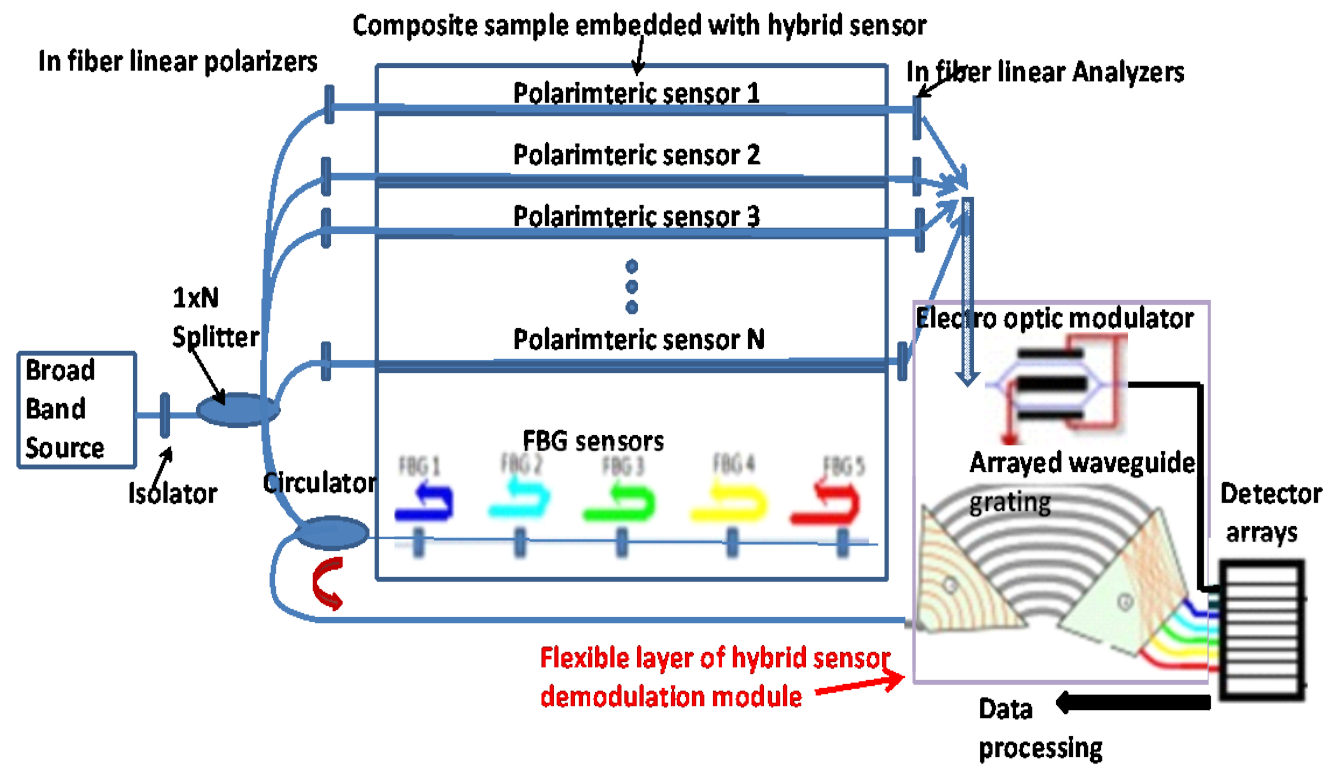

Figure 1. The schematic of the proposed flexible interrogation module

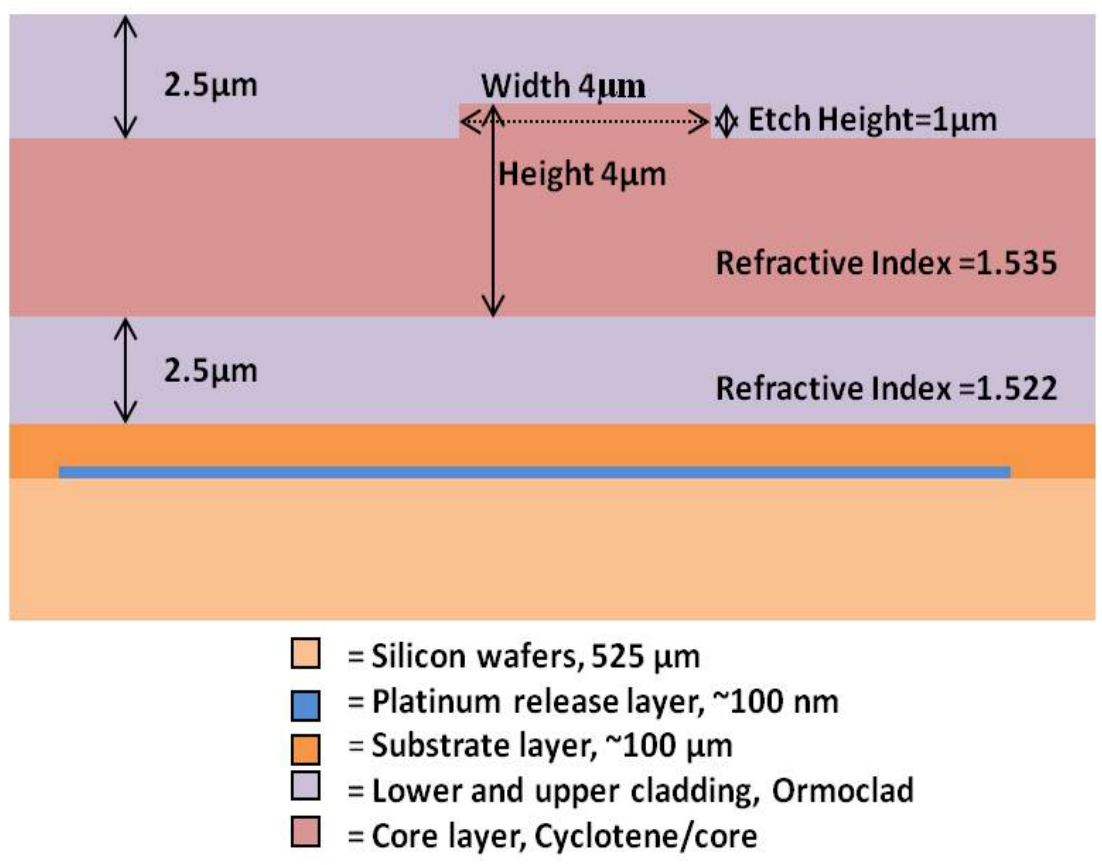

Figure 2. Schematic of fabrication of the proposed flexible interrogator 


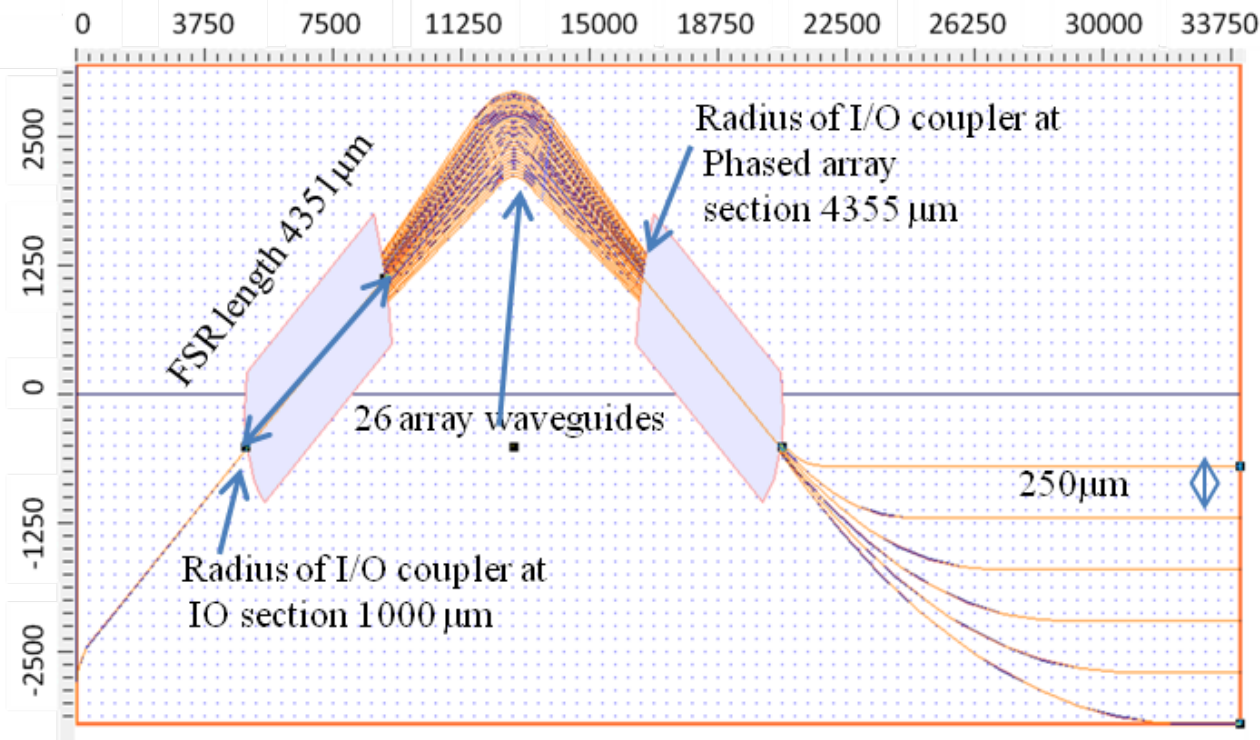

Figure 3 . The layout of the $1 \times 6$ channel AWG

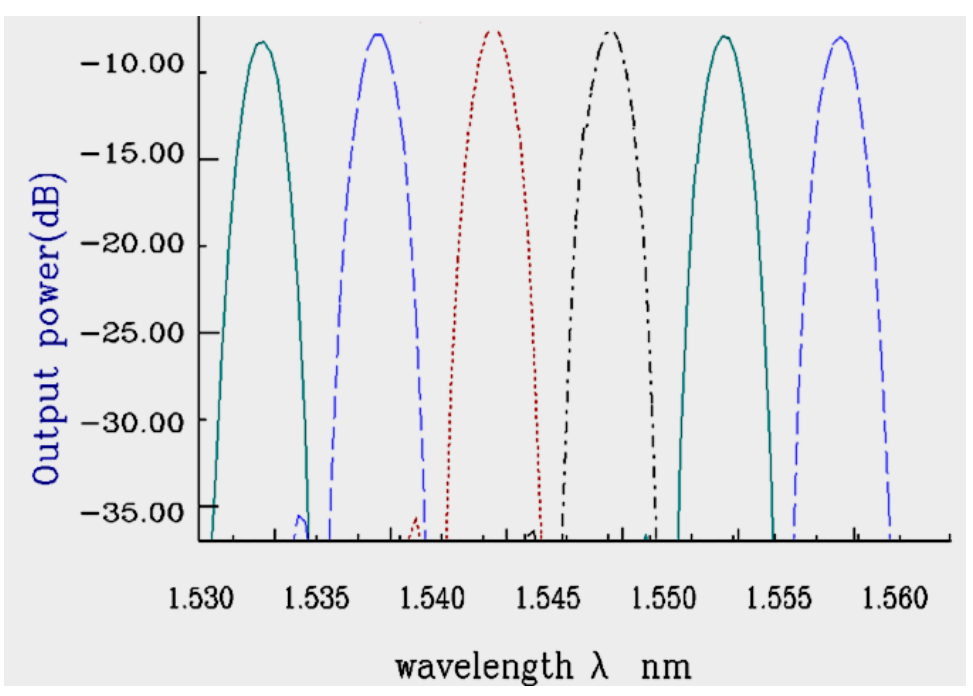

Figure 4. Simulated optical transmission of the AWG 


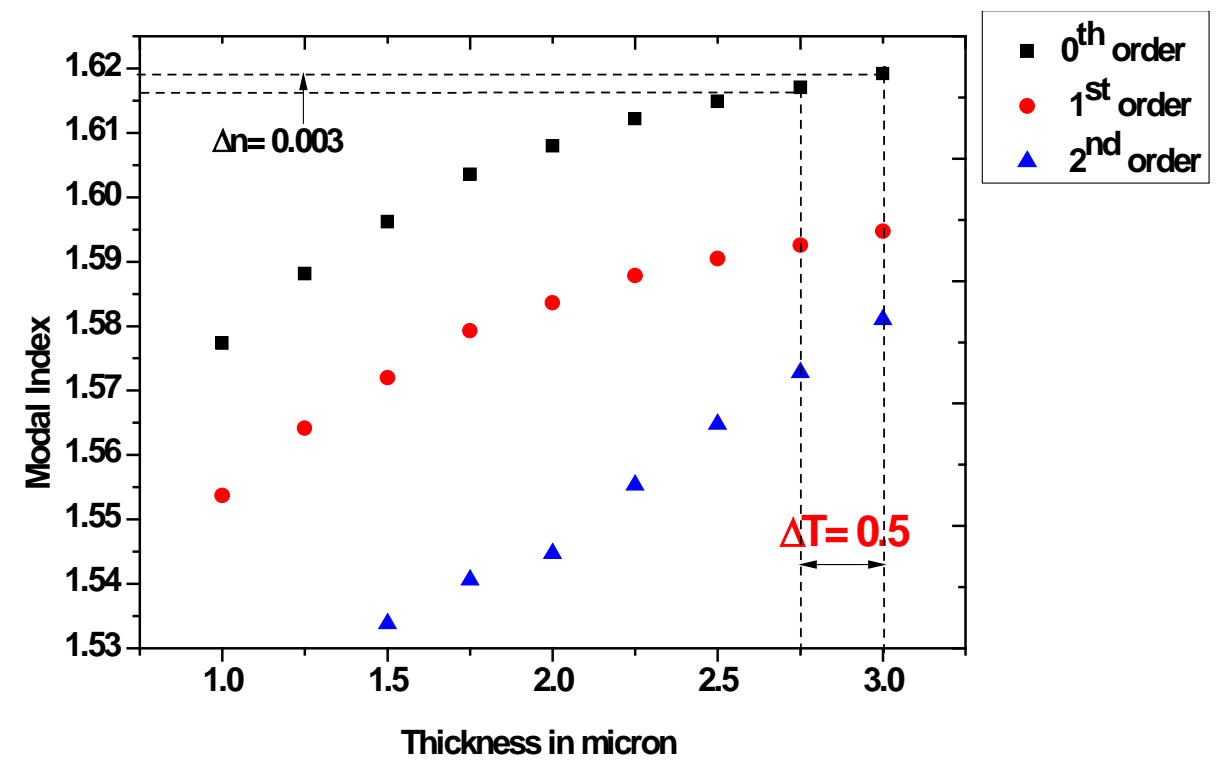

Figure. 5 Optimization rib height for single mode operation

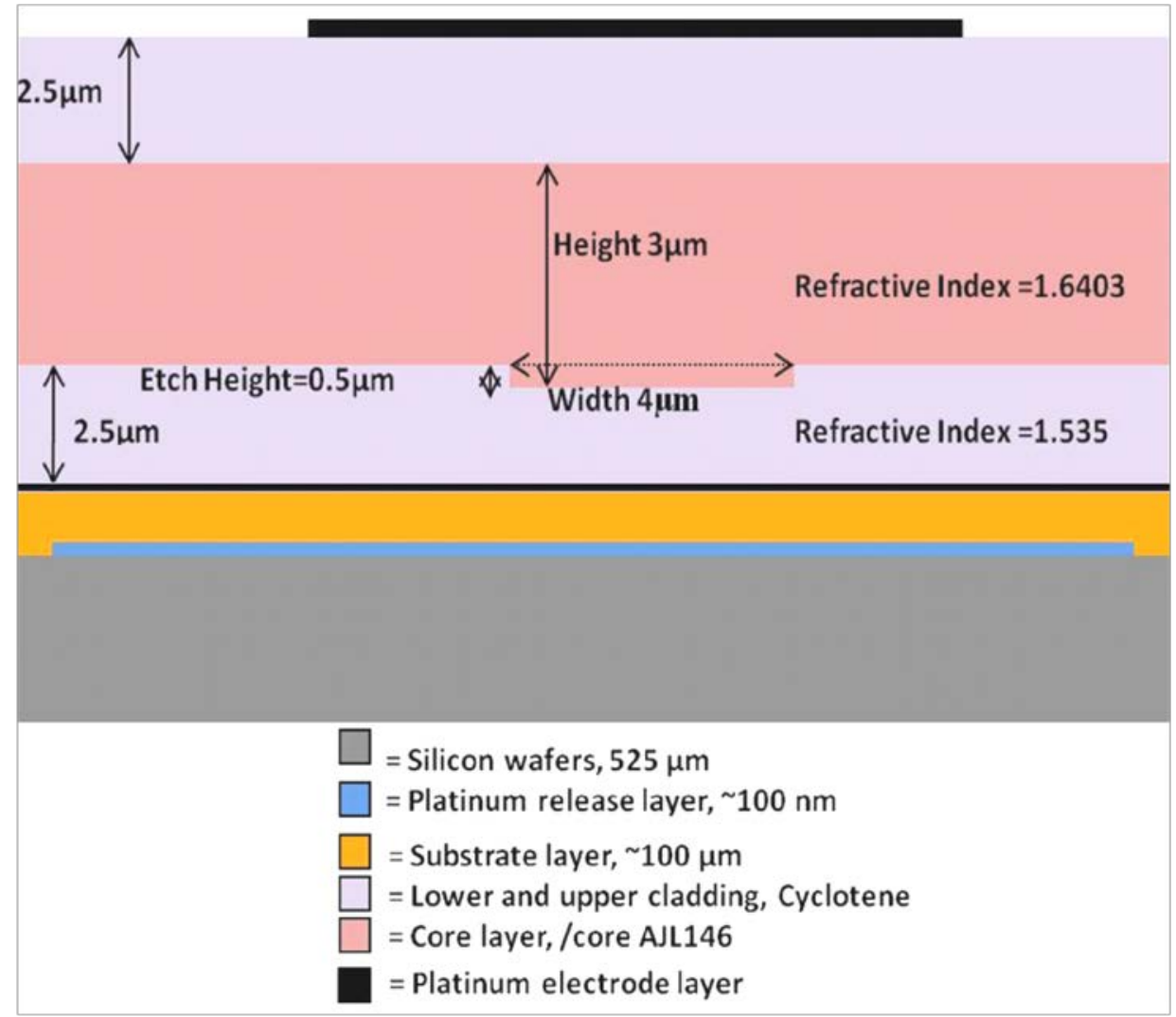

Figure. 6 Inverted Rib waveguide structure of MZI - EO modulator 


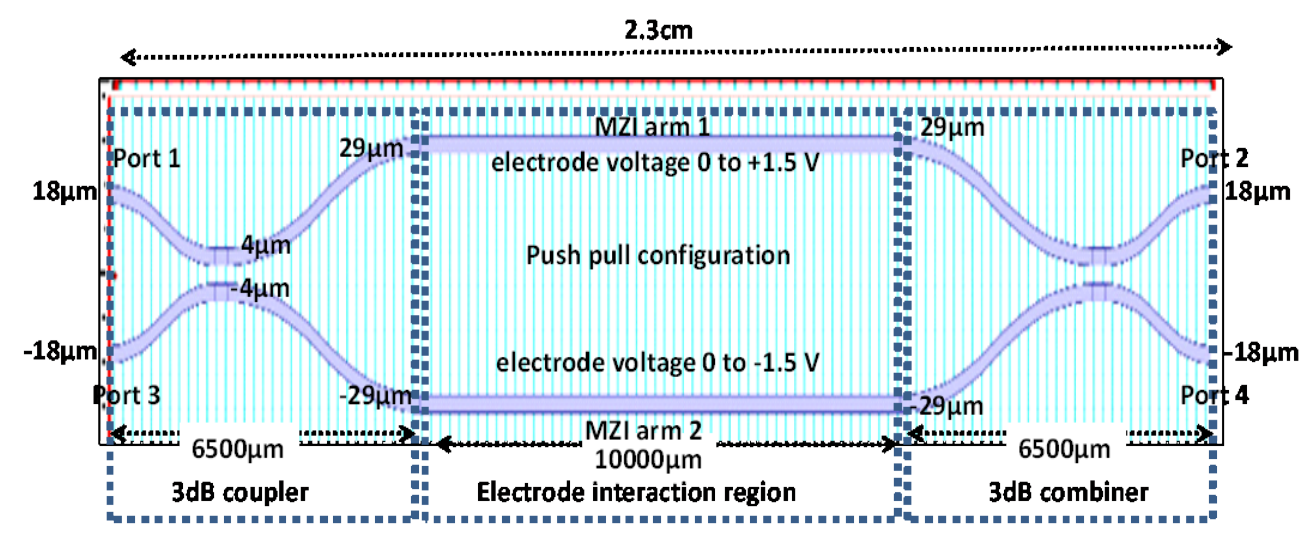

(a)

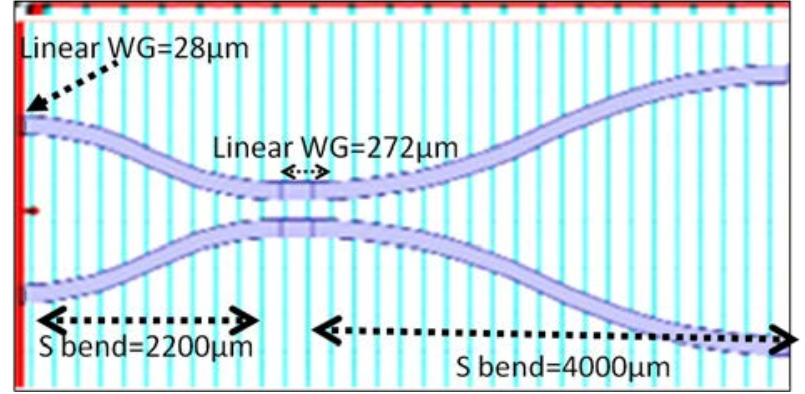

(b)

Figure. 7 (a) Layout of 2x2 MZI EO modulator with vertical offset values of $\mathrm{S}$ bend \& electrode voltages, (b) Layout and length of waveguides of $3 \mathrm{~dB}$ splitter/combiner

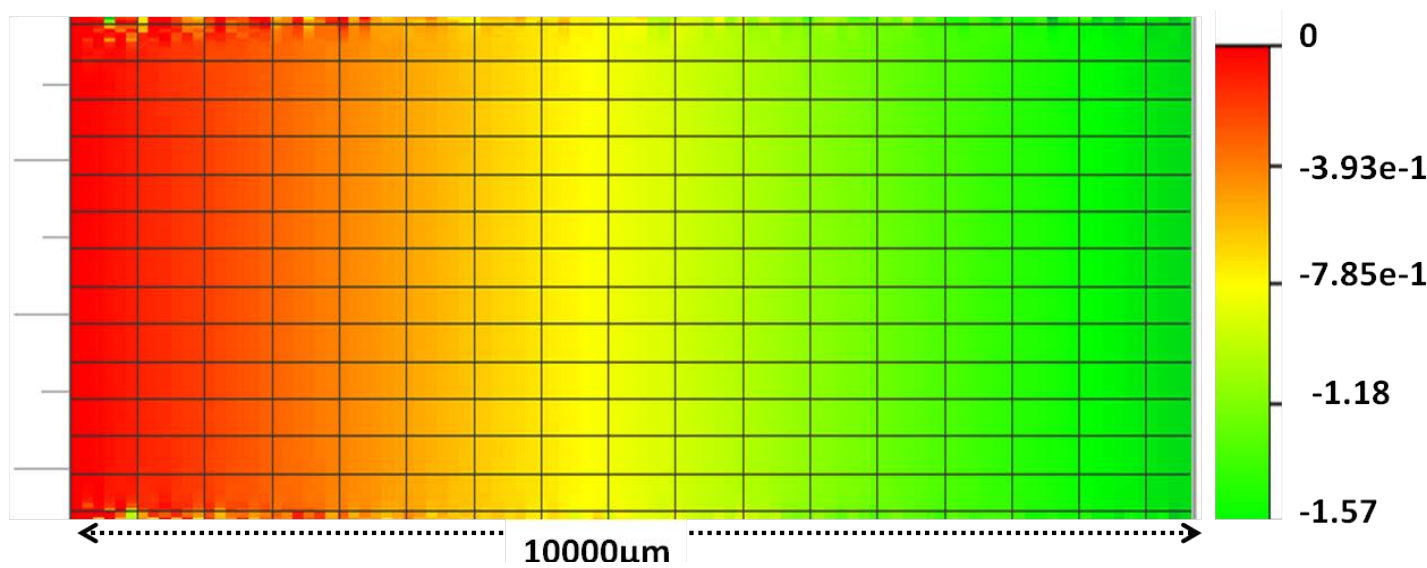

Figure. 8 Simulated phase change of $-\pi / 2$ for an electrode voltage of - 1.5 Volt and interaction length of $1 \mathrm{~cm}$ 


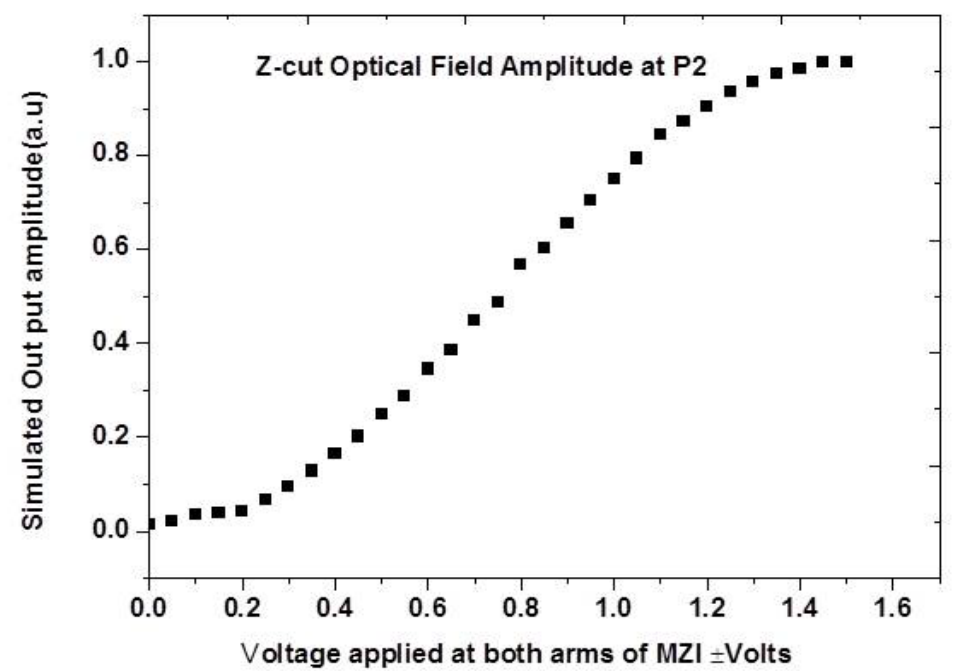

Figure. 9 Simulated optical field amplitude at P2 of MZI- EO device 

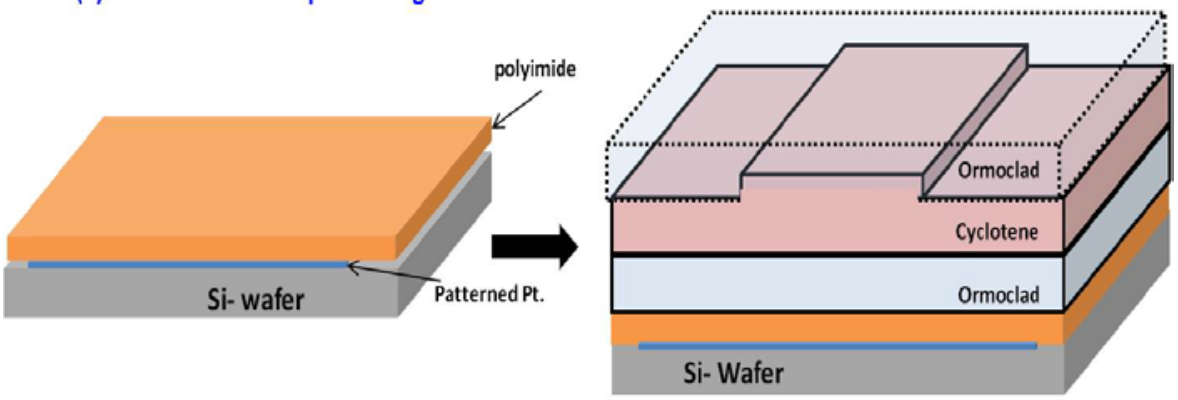

(d) Device lift off
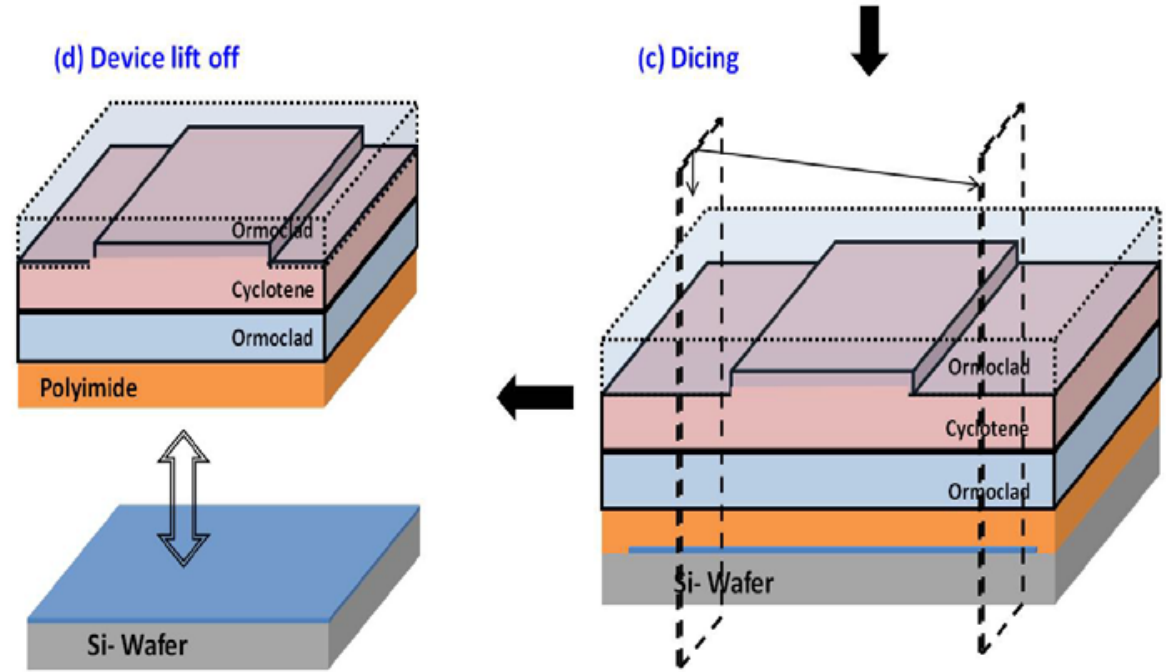

Figure. 10 The fabrication steps for the flexible AWG device (a) Releasing layer and flexible polyamide substrate patterning, (b) AWG device fabrication, (c) Dicing, and (d) Device lift-off. 
(a) Bottom electrode, \& flexible substrate

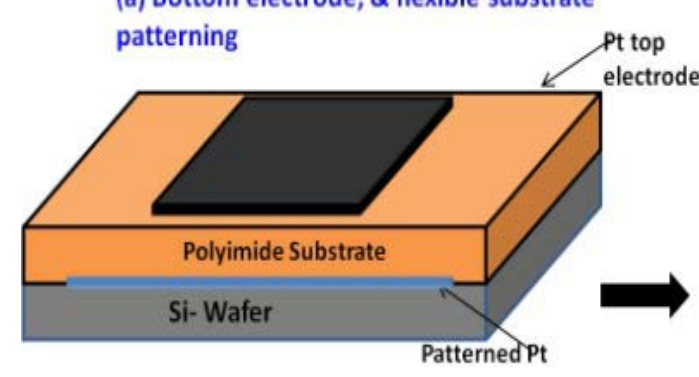

(d) Device lift off

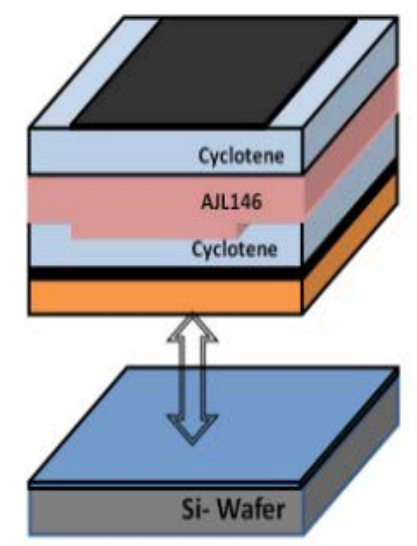

(b) EO device fabrication
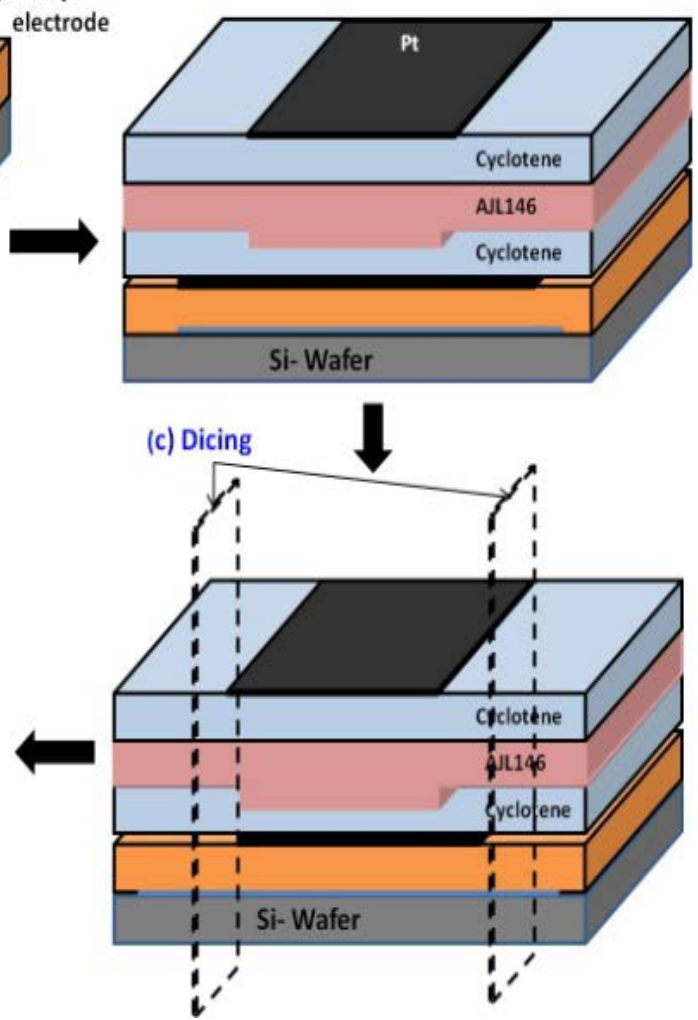

Figure. 11 The fabrication steps for the flexible MZI-EO device (a) Releasing layer and flexible polyimide substrate, bottom electrode patterning (b) MZI-EO device fabrication, (c) Dicing, and (d) Device lift-off.
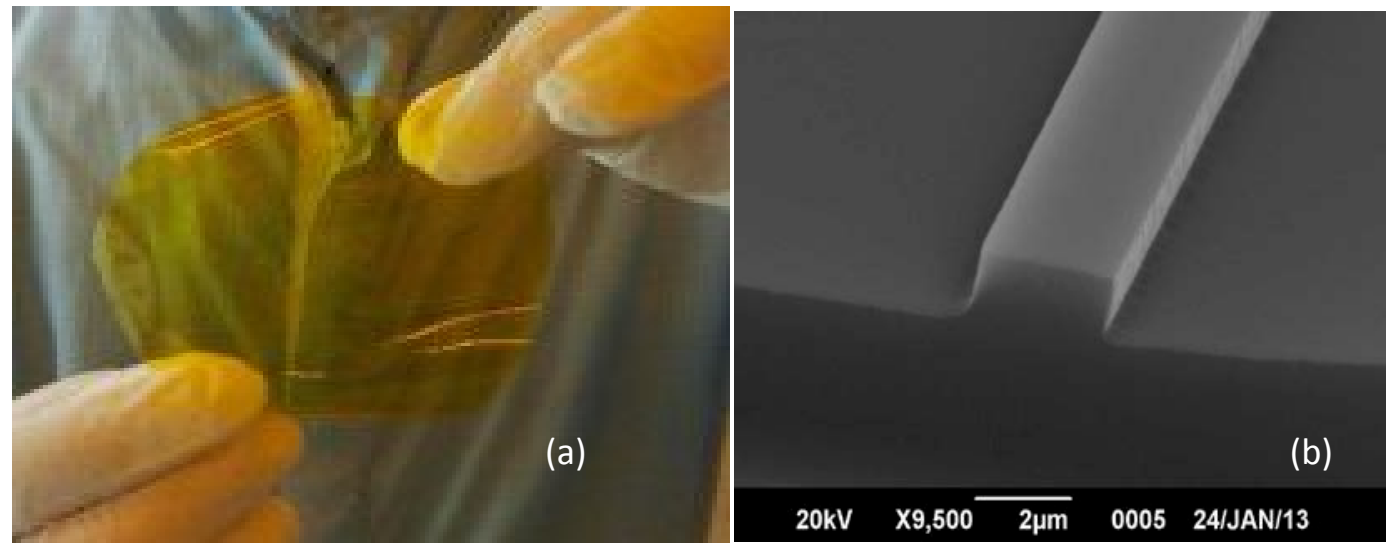

Figure. 12 (a) thin film type flexible interrogation module after fabrication. (b) SEM image of the rib waveguide structure of AWG device 
